\title{
Mean Square Heun's Method Convergent for Solving Random Differential Initial Value Problems of First Order
}

\author{
M. A. Sohaly \\ Department of Mathematics, Faculty of Science, Mansoura University, Mansoura, Egypt \\ Email: $\underline{m \text { stat2000@yahoo.com }}$
}

Received 23 September 2014; revised 20 October 2014; accepted 12 November 2014

Copyright (C) 2014 by author and Scientific Research Publishing Inc.

This work is licensed under the Creative Commons Attribution International License (CC BY).

http://creativecommons.org/licenses/by/4.0/

(c) (i)

Open Access

\begin{abstract}
This paper deals with the construction of Heun's method of random initial value problems. Sufficient conditions for their mean square convergence are established. Main statistical properties of the approximations processes are computed in several illustrative examples.
\end{abstract}

\section{Keywords}

Stochastic Partial Differential Equations, Mean Square Sense, Second Order Random Variable, Finite Difference Scheme

\section{Introduction}

Random differential equation (RDE), is an ordinary differential equation (ODE) with random inputs that can model unpredictable real-life behavior of any continuous system and they are important tools in modeling complex phenomena. They arise in many physics and engineering applications such as wave propagation, diffusion through heterogeneous random media. Additional examples can be found in materials science, chemistry, biology, and other areas. However, reaching a solution of these equations in a closed form is not always possible or even easy. Due to the complex nature of RDEs, numerical simulations play an important role in studying this class of DEs. For this reason, few numerical and analytical methods have been developed for simulating RDEs. Random solutions are always studied in terms of their statistical measures.

This paper is interested in studying the following random differential initial value problem (RIVP) of the form:

$$
\frac{\mathrm{d} X(t)}{\mathrm{d} t}=f(t, X(t)), \quad X\left(t_{0}\right)=X_{0} .
$$


Randomness may exist in the initial value or in the differential operator or both. In [1] [2], the authors discussed the general order conditions and a global convergence proof was given for stochastic Runge-Kutta methods applied to stochastic ordinary differential equations (SODEs) of Stratonovich type. In [3] [4], the authors discussed the random Euler method and the conditions for the mean square convergence of this problem. In [5], the authors considered a very simple adaptive algorithm, based on controlling only the drift component of a time step but if the drift was not linearly bounded, then explicit fixed time step approximations, such as the EulerMaruyama scheme, may fail to be ergodic. Platen, E. [6] discussed discrete time strong and weak approximation methods that were suitable for different applications. [12] discussed the mean square convergent Euler and Runge Kutta methods for random initial value problem. Other numerical methods are discussed in [7]-[12].

In this paper the random Heun's method is used to obtain an approximate solution for Equation (1). This paper is organized as follows. In Section 2, some important preliminaries are discussed. In Section 3, random differential equations are discussed. In Section 4, the convergence of random Heun's method is discussed. Section 5 presents the solution of numerical example of first order random differential equation using random Heun's method showing the convergence of the numerical solutions to the exact ones (if possible). The general conclusions are presented in Section 6.

\section{Preliminaries}

Definition 1 [13]. Let us consider the properties of a class of real r.v.'s $X_{1}, X_{2}, \cdots, X_{n}$ whose second moments, $E\left\{X_{1}^{2}\right\}, E\left\{X_{2}^{2}\right\}, \cdots$ are finite. In this case, they are called "second order random variables", (2.r.v's).

\section{The Convergence in Mean Square [13]}

A sequence of r.v's $\left\{X_{n}\right\}$ converges in mean square (m.s) to a random variable $X$ if: $\lim _{n \rightarrow \infty}\left\|X_{n}-X\right\|=0$ i.e. $X_{n} \stackrel{\text { m.s }}{\longrightarrow} X$ or $\lim _{n \rightarrow \infty} X_{n}=X$ where l.i.m is the limit in mean square sense.

\section{Random Initial Value Problem (RIVP)}

If we have the random differential equation

$$
\dot{X}(t)=f(X(t), t), \quad t \in T=\left[t_{0}, t_{1}\right], \quad X\left(t_{0}\right)=X_{0}
$$

where $X_{0}$ is a random variable, and the unknown $X(t)$ as well as the right-hand side $f(X, t)$ are stochastic processes defined on the same probability space $(\Omega, F, P)$, are powerful tools to model real problems with uncertainty.

Definition 2 [6] [7].

- Let $g: T \rightarrow L_{2}$ is an m.s. bounded function and let $h>0$ then The "m.s. modulus of continuity of $g$ " is the function

$$
W(g, h)=\sup _{\left|t-t^{*}\right| \leq h}\left\|g(t)-g\left(t^{*}\right)\right\|, \quad t, t^{*} \in T .
$$

- The function $g$ is said to be m.s. uniformly continuous in $T$ if:

$$
\lim _{h \rightarrow 0} W(g, h)=0 .
$$

Note that: (The limit depend on $h$ because $g$ is defined at every $t$ so we can write $W(g, h)=W(h)$ ).

In the problem (2) that we discusses we find that the convergence of this problem is depend on the right hand side (i.e. $f(X(t), t)$ ) then we want to apply the previous definition on $f(X(t), t)$ hence:

Let $f(X(t), t)$ be defined on $S \times T$ where $S$ is bounded set in $L_{2}$ Then we say that $f$ is "randomly bounded uniformly continuous” in $S$, if $\lim _{h \rightarrow 0} W(f(x,), h)=$.0 (note that: $W(f(X(), h))=.W(h)$ ).

\section{A Random Mean Value Theorem for Stochastic Processes}

The aim of this section is to establish a relationship between the increment $X(t)-X\left(t_{0}\right)$ of a 2-s.p. and its m.s. 
derivative $\dot{X}(\xi)$ for some $\xi$ lying in the interval $\left[t_{0}, t\right]$ for $t>t_{0}$. This result will be used in the next section to prove the convergence of the random Heun's method discussed.

Lemma (3.2) [6] [7].

Let $Y(t)$ is a 2-s.p., m.s. continuous on interval $T=\left[t_{0}, t_{1}\right]$. Then, there exists $\xi \in\left[t_{0}, t_{1}\right]$ such that:

$$
\int_{t_{0}}^{t} Y(s) \mathrm{d} s=Y(\xi)\left(t-t_{0}\right), \quad t_{0}<t<t_{1} .
$$

Theorem (3.3) [6] [7].

Let $X(s)$ be a m.s. differentiable 2-s.p. in $] t_{0}, t_{1}\left[\right.$ and m.s. continuous in $T=\left[t_{0}, t_{1}\right]$. Then, there exists $\xi \in\left[t_{0}, t_{1}\right]$ such that:

$$
X(t)-X\left(t_{0}\right)=\dot{X}(\xi)\left(t-t_{0}\right)
$$

\section{The Convergence of Random Heun's Scheme}

In this section we are interested in the mean square convergence, in the fixed station sense, of the random Heun's method defined by:

$$
X_{n+1}=X_{n}+\frac{h}{2}\left[f\left(X_{n}, t_{n}\right)+f\left(X_{n}+h f\left(X_{n}, t_{n}\right), t_{n+1}\right)\right], \quad X\left(t_{0}\right)=X_{0}, \quad n \geq 0
$$

where $X_{n}$ and $f\left(X_{n}, t_{n}\right)$ are 2-r.v.'s, $h=t_{n}-t_{n-1}, t_{n}=t_{0}+n h$ and $f: S \times T \rightarrow L_{2}, S \subset L_{2}$ satisfies the following conditions:

C1: $f(X, t)$ is randomly bounded uniformly continuous.

C2: $f(X, t)$ satisfies the m.s. Lipschitz condition:

$$
\|f(x, t)-f(y, t)\| \leq k(t)\|x-y\| \text { where }: \int_{t_{0}}^{t_{1}} k(t) \leq \infty .
$$

Note that under hypothesis C1 and C2, we are interested in the m.s. convergence to zero of the error:

$$
e_{n}=X_{n}-X(t)
$$

where $X(t)$ is the theoretical solution 2-s.p. of the problem (2), $t=t_{n}=t_{0}+n h$.

Taking into account (2), and Th (3.3), one gets, since from (2) we have at $t=t_{\xi}$ then: $\dot{X}\left(t_{\xi}\right)=f\left(x\left(t_{\xi}\right), t_{\xi}\right)$. (Note: $\xi \in\left[t_{0}, t_{1}\right]$ and we can use $\xi$ instead of $t_{\xi}$.)

And from Th (3.3) at $t=t_{\xi}$ then we obtain:

$$
X\left(t_{\xi}\right)-X\left(t_{0}\right)=\dot{X}(t)\left(t_{\xi}-t_{0}\right) \Rightarrow X\left(t_{\xi}\right)-X\left(t_{0}\right)=f\left(X\left(t_{\xi}, t_{\xi}\right)\right)\left(t_{\xi}-t_{0}\right) .
$$

Note that we deal with the interval $\left(t_{n}, t_{n+1}\right) \quad \ni t_{\xi} \in\left(t_{n}, t_{n+1}\right)$ and hence $t_{0}$ was the starting in the problem (2) and here $t_{n}$ is the starting and since Heun's method deal with solution depend on previous solution and if we have $X\left(t_{n}\right)$ instead of $X\left(t_{0}\right)$ then we can use $X\left(t_{n+1}\right)$ instead of $X\left(t_{\xi}\right)$ hence the final form of the problem (2) is:

$$
X\left(t_{n+1}\right)=X\left(t_{n}\right)+h f\left(X\left(t_{\xi}, t_{\xi}\right)\right) \text {, for some } t_{\xi} \in\left(t_{n}, t_{n+1}\right) .
$$

Now we have the solution of problem (2) is: $X\left(t_{n}\right)$.

At $t=t_{n}$ then $X\left(t_{n}\right)=X(t)$ and the solution of Heun's method (4) is: $X_{n}$.

Then we can define the error:

$$
e_{n}=X_{n}-X(t) \text {. }
$$

By (4) and (7) it follows that:

$$
\begin{aligned}
e_{n+1}=X_{n+1}-X\left(t_{n+1}\right)= & X_{n}+\frac{h}{2}\left[f\left(X_{n}, t_{n}\right)+f\left(X_{n}+h f\left(X_{n}, t_{n}\right), t_{n+1}\right)\right] \\
& -X\left(t_{n}\right)-\frac{h}{2} f\left(X\left(t_{\xi}\right), t_{\xi}\right)-\frac{h}{2} f\left(X\left(t_{\xi}\right), t_{\xi}\right) .
\end{aligned}
$$

Then we obtain: 


$$
e_{n+1}=X_{n}-X\left(t_{n}\right)+\frac{h}{2}\left\{f\left(X_{n}, t_{n}\right)-f\left(X\left(t_{\xi}\right), t_{\xi}\right)\right\}+\frac{h}{2}\left\{f\left(X_{n}+h f\left(X_{n}, t_{n}\right), t_{n+1}\right)-f\left(X\left(t_{\xi}\right), t_{\xi}\right)\right\} .
$$

By taking the norm for the two sides:

$$
\begin{aligned}
\left\|e_{n+1}\right\| & =\left\|X_{n}-X\left(t_{n}\right)+\frac{h}{2}\left\{f\left(X_{n}, t_{n}\right)-f\left(X\left(t_{\xi}\right), t_{\xi}\right)\right\}+\frac{h}{2}\left\{f\left(X_{n}+h f\left(X_{n}, t_{n}\right), t_{n+1}\right)-f\left(X\left(t_{\xi}\right), t_{\xi}\right)\right\}\right\| \\
& \leq\left\|X_{n}-X\left(t_{n}\right)\right\|+\frac{h}{2}\left\|f\left(X\left(t_{\xi}\right), t_{\xi}\right)-f\left(X_{n}, t_{n}\right)\right\|+\frac{h}{2}\left\|f\left(X_{n}+h f\left(X_{n}, t_{n}\right), t_{n+1}\right)-f\left(X\left(t_{\xi}\right), t_{\xi}\right)\right\| .
\end{aligned}
$$

Since:

$$
\begin{aligned}
& \left\|f\left(X\left(t_{\xi}\right), t_{\xi}\right)-f\left(X_{n}, t_{n}\right)\right\| \\
& =\left\|f\left(X\left(t_{\xi}\right), t_{\xi}\right)-f\left(X\left(t_{\xi}\right), t_{n}\right)+f\left(X\left(t_{\xi}\right), t_{n}\right)+f\left(X\left(t_{n}\right), t_{n}\right)-f\left(X\left(t_{n}\right), t_{n}\right)-f\left(X_{n}, t_{n}\right)\right\| \\
& \leq\left\|f\left(X\left(t_{\xi}\right), t_{\xi}\right)-f\left(X\left(t_{\xi}\right), t_{n}\right)\right\|+\left\|f\left(X\left(t_{\xi}\right), t_{n}\right)-f\left(X\left(t_{n}\right), t_{n}\right)\right\|+\left\|f\left(X\left(t_{n}\right), t_{n}\right)-f\left(X_{n}, t_{n}\right)\right\| .
\end{aligned}
$$

Since the theoretical solution $X(t)$ is m.s. bounded in $\left[t_{0}, t_{1}\right], \sup _{t_{0} \leq t \leq t_{1}}\|X(t)\| \leq M<\infty$ and under hypothesis C1, C2, we have:

- $\left\|f\left(X\left(t_{\xi}\right), t_{\xi}\right)-f\left(X\left(t_{\xi}\right), t_{n}\right)\right\|=w(h)$.

- $\left\|f\left(X\left(t_{\xi}\right), t_{n}\right)-f\left(X\left(t_{n}\right), t_{n}\right)\right\| \leq k\left(t_{n}\right) M h$

where $k\left(t_{n}\right)$ is Lipschitz constant (from C2) and:

From Th (3.3) we have $X(t)-X\left(t_{0}\right)=\dot{X}(\xi)\left(t-t_{0}\right)$ and note that the two points are $X\left(t_{\xi}\right)$ and $X\left(t_{n}\right)$ in (10) then:

$$
\left\|X\left(t_{\xi}\right)-X\left(t_{n}\right)\right\|=\|\dot{X}(\xi)\|\left|t_{\xi}-t_{n}\right| \leq M h
$$

where: $\left|t_{\xi}-t_{n}\right|=h$ and $M=\sup _{t_{0} \leq t \leq t_{1}}|| \dot{X}(t) \|$.

- $\left\|f\left(X\left(t_{n}\right), t_{n}\right)-f\left(X_{n}, t_{n}\right)\right\| \leq k\left(t_{n}\right)\left\|X\left(t_{n}\right)-X_{n}\right\|=k\left(t_{n}\right)\left\|e_{n}\right\|$.

Then by substituting in (9) we have:

$$
\left\|f\left(X\left(t_{\xi}\right), t_{\xi}\right)-f\left(X_{n}, t_{n}\right)\right\| \leq w(h)+k\left(t_{n}\right) M h+k\left(t_{n}\right)\left\|e_{n}\right\| .
$$

And another term:

$$
\begin{aligned}
\| & f\left(X_{n}+h f\left(X_{n}, t_{n}\right), t_{n+1}\right)-f\left(X\left(t_{\xi}\right), t_{\xi}\right)\|=\| f\left(X\left(t_{\xi}\right), t_{\xi}\right)-f\left(X_{n}+h f\left(X_{n}, t_{n}\right), t_{n+1}\right) \| \\
= & \| f\left(X\left(t_{\xi}\right), t_{\xi}\right)-f\left(X\left(t_{\xi}\right), t_{n+1}\right)+f\left(X\left(t_{\xi}\right), t_{n+1}\right)+f\left(X\left(t_{n}\right), t_{n+1}\right) \\
& -f\left(X\left(t_{n}\right), t_{n+1}\right)-f\left(X_{n}+h f\left(X_{n}, t_{n}\right), t_{n+1}\right) \| \\
\leq & \left\|f\left(X\left(t_{\xi}\right), t_{\xi}\right)-f\left(X\left(t_{\xi}\right), t_{n+1}\right)\right\|+\left\|f\left(X\left(t_{\xi}\right), t_{n+1}\right)-f\left(X\left(t_{n}\right), t_{n+1}\right)\right\| \\
& +\left\|f\left(X\left(t_{n}\right), t_{n+1}\right)-f\left(X_{n}+h f\left(X_{n}, t_{n}\right), t_{n+1}\right)\right\| \\
\leq & w(h)+k\left(t_{n+1}\right) M h+k\left(t_{n+1}\right)\left[\left\|e_{n}\right\|-h M\right] .
\end{aligned}
$$

Since:

- $\left\|f\left(X\left(t_{\xi}\right), t_{\xi}\right)-f\left(X\left(t_{\xi}\right), t_{n+1}\right)\right\| \leq w(h)$.

- $\left\|f\left(X\left(t_{\xi}\right), t_{n+1}\right)-f\left(X\left(t_{n}\right), t_{n+1}\right)\right\| \leq k\left(t_{n+1}\right) M h$ 
where $k\left(t_{n}\right)$ is Lipschitz constant (from C2) and:

From Th (3.3) we have $X(t)-X\left(t_{0}\right)=\dot{X}(\xi)\left(t-t_{0}\right)$ and note that the two points are $X\left(t_{\xi}\right)$ and $X\left(t_{n}\right)$ in (10) then we have:

$$
\left\|X\left(t_{\xi}\right)-X\left(t_{n}\right)\right\|=\|\dot{X}(\xi)\|\left|t_{\xi}-t_{n}\right| \leq M h
$$

where $\left|t_{\xi}-t_{n}\right|=h$ and $M=\sup _{t_{0} \leq t \leq t_{1}}\|\dot{X}(t)\|$.

And the last term:

$$
\begin{aligned}
& \left\|f\left(X\left(t_{n}\right), t_{n+1}\right)-f\left(X_{n}+h f\left(X_{n}, t_{n}\right), t_{n+1}\right)\right\| \leq k\left(t_{n+1}\right)\left\|X\left(t_{n}\right)-X_{n}-h f\left(X_{n}, t_{n}\right)\right\| \\
& \leq k\left(t_{n+1}\right)\left[\left\|X\left(t_{n}\right)-X_{n}\right\|-\left\|h f\left(X_{n}, t_{n}\right)\right\|\right]=k\left(t_{n+1}\right)\left[\left\|e_{n}\right\|-h M\right] .
\end{aligned}
$$

Then by substituting in (8) we have:

$$
\begin{aligned}
&\left\|e_{n+1}\right\| \leq\left\|e_{n}\right\|+\frac{h}{2}\left[w(h)+k\left(t_{n}\right) M h+k\left(t_{n}\right)\left\|e_{n}\right\|\right]+\frac{h}{2}\left[w(h)+k\left(t_{n+1}\right) M h+k\left(t_{n+1}\right)\left[\left\|e_{n}\right\|-h M\right]\right] \\
&=\left\|e_{n}\right\|\left(1+\frac{h}{2} k\left(t_{n}\right)+k\left(t_{n+1}\right)\right)+\frac{h}{2}\left[2 w(h)+h k\left(t_{n}\right) M+h k\left(t_{n+1}\right) M-K\left(t_{n+1}\right) h M\right] \\
& \leq\left\{\left\|e_{n-1}\right\|\left(1+\frac{h}{2} k\left(t_{n-1}\right)+k\left(t_{n}\right)\right)+\frac{h}{2}\left[2 w(h)+h k\left(t_{n-1}\right) M+h k\left(t_{n}\right) M-K\left(t_{n}\right) h M\right]\right\} \\
& \times\left\{\left(1+\frac{h}{2} k\left(t_{n}\right)+k\left(t_{n+1}\right)\right)\right\}+\frac{h}{2}\left[2 w(h)+h k\left(t_{n}\right) M+h k\left(t_{n+1}\right) M-K\left(t_{n+1}\right) h M\right] \\
&=\left\|e_{n-1}\right\|\left(1+\frac{h}{2} k\left(t_{n-1}\right)+k\left(t_{n}\right)\right)\left(1+\frac{h}{2} k\left(t_{n}\right)+k\left(t_{n+1}\right)\right)+\frac{h}{2}\left[2 w(h)+h k\left(t_{n-1}\right) M+h k\left(t_{n}\right) M-K\left(t_{n}\right) h M\right] \\
& \times\left(1+\frac{h}{2} k\left(t_{n}\right)+k\left(t_{n+1}\right)\right)+\frac{h}{2}\left[2 w(h)+h k\left(t_{n}\right) M+h k\left(t_{n+1}\right) M-K\left(t_{n+1}\right) h M\right] \\
& \leq {\left[\left\{\left\|e_{n-2}\right\|\left(1+\frac{h}{2} k\left(t_{n-2}\right)+k\left(t_{n-1}\right)\right)+\frac{h}{2}\left[2 w(h)+h k\left(t_{n-2}\right) M+h k\left(t_{n-1}\right) M-K\left(t_{n-1}\right) h M\right]\right\}\right.} \\
&\left.\times\left(1+\frac{h}{2} k\left(t_{n-1}\right)+k\left(t_{n}\right)\right)+\frac{h}{2}\left[2 w(h)+h k\left(t_{n-1}\right) M+h k\left(t_{n}\right) M-K\left(t_{n}\right) h M\right]\right] \\
& \times\left(1+\frac{h}{2} k\left(t_{n}\right)+k\left(t_{n+1}\right)\right)+\frac{h}{2}\left[2 w(h)+h k\left(t_{n}\right) M+h k\left(t_{n+1}\right) M-K\left(t_{n+1}\right) h M\right] \\
&=\left\|e_{n-2}\right\|\left(1+\frac{h}{2} k\left(t_{n-2}\right)+k\left(t_{n-1}\right)\right)\left(1+\frac{h}{2} k\left(t_{n-1}\right)+k\left(t_{n}\right)\right)\left(1+\frac{h}{2} k\left(t_{n}\right)+k\left(t_{n+1}\right)\right) \\
&\left.+\frac{h}{2}\left[2 w(h)+h k\left(t_{n-2}\right) M+h k\left(t_{n-1}\right) M-K\left(t_{n-1}\right) h M\right]\left(1+\frac{h}{2} k\left(t_{n-1}\right)+k\left(t_{n}\right)\right)\right)\left(1+\frac{h}{2} k\left(t_{n}\right)+k\left(t_{n+1}\right)\right) \\
&+\frac{h}{2}\left[2 w(h)+h k\left(t_{n-1}\right) M+h k\left(t_{n}\right) M-K\left(t_{n}\right) h M\right]\left(1+\frac{h}{2} k\left(t_{n}\right)+k\left(t_{n+1}\right)\right) \\
&+\frac{h}{2}\left[2 w(h)+h k\left(t_{n}\right) M+h k\left(t_{n+1}\right) M-K\left(t_{n+1}\right) h M\right] \\
& \leq {\left[\left[\left\{\left\|e_{n-3}\right\|\left(1+\frac{h}{2} k\left(t_{n-3}\right)+k\left(t_{n-2}\right)\right)+\frac{h}{2}\left[2 w(h)+h k\left(t_{n-3}\right) M+h k\left(t_{n-2}\right) M-K\left(t_{n-2}\right) h M\right]\right\}\right.\right.} \\
&\left.\times\left(1+\frac{h}{2} k\left(t_{n-2}\right)+k\left(t_{n-1}\right)\right)+\frac{h}{2}\left[2 w(h)+h k\left(t_{n-2}\right) M+h k\left(t_{n-1}\right) M-K\left(t_{n-1}\right) h M\right]\right] \\
&\left.\left.\times\left(t_{n-1}\right)+k\left(t_{n}\right)\right)+\frac{h}{2}\left[2 w(h)+h k\left(t_{n-1}\right) M+h k\left(t_{n}\right) M-K\left(t_{n}\right) h M\right]\right] \\
&
\end{aligned}
$$




$$
\begin{aligned}
& \times\left(1+\frac{h}{2} k\left(t_{n}\right)+k\left(t_{n+1}\right)\right)+\frac{h}{2}\left[2 w(h)+h k\left(t_{n}\right) M+h k\left(t_{n+1}\right) M-K\left(t_{n+1}\right) h M\right] \\
= & \left\|e_{n-3}\right\|\left(1+\frac{h}{2} k\left(t_{n-3}\right)+k\left(t_{n-2}\right)\right)\left(1+\frac{h}{2} k\left(t_{n-2}\right)+k\left(t_{n-1}\right)\right)\left(1+\frac{h}{2} k\left(t_{n-1}\right)+k\left(t_{n}\right)\right)\left(1+\frac{h}{2} k\left(t_{n}\right)+k\left(t_{n+1}\right)\right) \\
& +\frac{h}{2}\left[2 w(h)+h k\left(t_{n-2}\right) M+h k\left(t_{n-1}\right) M-K\left(t_{n-1}\right) h M\right]\left(1+\frac{h}{2} k\left(t_{n-2}\right)+k\left(t_{n-1}\right)\right) \\
& \times\left(1+\frac{h}{2} k\left(t_{n-1}\right)+k\left(t_{n}\right)\right)\left(1+\frac{h}{2} k\left(t_{n}\right)+k\left(t_{n+1}\right)\right)+\frac{h}{2}\left[2 w(h)+h k\left(t_{n-1}\right) M+h k\left(t_{n}\right) M-K\left(t_{n}\right) h M\right] \\
& \times\left(1+\frac{h}{2} k\left(t_{n-1}\right)+k\left(t_{n}\right)\right)\left(1+\frac{h}{2} k\left(t_{n}\right)+k\left(t_{n+1}\right)\right)+\frac{h}{2}\left[2 w(h)+h k\left(t_{n}\right) M+h k\left(t_{n+1}\right) M-K\left(t_{n+1}\right) h M\right] \\
& \times\left(1+\frac{h}{2} k\left(t_{n}\right)+k\left(t_{n+1}\right)\right)+\frac{h}{2}\left[2 w(h)+h k\left(t_{n-1}\right) M+h k\left(t_{n}\right) M-K\left(t_{n}\right) h M\right] .
\end{aligned}
$$

Finally, we have:

$$
\begin{aligned}
& \left\|e_{n+1}\right\| \leq\left\|e_{0}\right\|\left(1+\frac{h}{2} k\left(t_{0}\right)+k\left(t_{n-(n-1)}\right)\right)\left(1+\frac{h}{2} k\left(t_{n-(n-1)}\right)+k\left(t_{n-(n-2)}\right)\right)\left(1+\frac{h}{2} k\left(t_{n-(n-2)}\right)+k\left(t_{n-(n-3)}\right)\right) \cdots \\
& \times\left(1+\frac{h}{2} k\left(t_{n-1}\right)+k\left(t_{n}\right)\right)\left(1+\frac{h}{2} k\left(t_{n}\right)+k\left(t_{n+1}\right)\right)+\frac{h}{2}\left[2 w(h)+h k\left(t_{n-2}\right) M+h k\left(t_{n-1}\right) M-K\left(t_{n-1}\right) h M\right] \\
& \times\left(1+\frac{h}{2} k\left(t_{n-(n-1)}\right)+k\left(t_{n-(n-2)}\right)\right)\left(1+\frac{h}{2} k\left(t_{n-(n-2)}\right)+k\left(t_{n-(n-3)}\right)\right) \cdots\left(1+\frac{h}{2} k\left(t_{n-1}\right)+k\left(t_{n}\right)\right) \\
& \times\left(1+\frac{h}{2} k\left(t_{n}\right)+k\left(t_{n+1}\right)\right)\left(1+\frac{h}{2} k\left(t_{n-2}\right)+k\left(t_{n-1}\right)\right)\left(1+\frac{h}{2} k\left(t_{n-1}\right)+k\left(t_{n}\right)\right)\left(1+\frac{h}{2} k\left(t_{n}\right)+k\left(t_{n+1}\right)\right) \\
& +\frac{h}{2}\left[2 w(h)+h k\left(t_{n-1}\right) M+h k\left(t_{n}\right) M-K\left(t_{n}\right) h M\right]\left(1+\frac{h}{2} k\left(t_{n-(n-2)}\right)+k\left(t_{n-(n-3)}\right)\right) \cdots\left(1+\frac{h}{2} k\left(t_{n-1}\right)+k\left(t_{n}\right)\right) \\
& \times\left(1+\frac{h}{2} k\left(t_{n}\right)+k\left(t_{n+1}\right)\right)\left(1+\frac{h}{2} k\left(t_{n-2}\right)+k\left(t_{n-1}\right)\right)\left(1+\frac{h}{2} k\left(t_{n-1}\right)+k\left(t_{n}\right)\right)\left(1+\frac{h}{2} k\left(t_{n}\right)+k\left(t_{n+1}\right)\right)+\cdots \\
& +\frac{h}{2}\left[2 w(h)+h k\left(t_{n}\right) M+h k\left(t_{n+1}\right) M-K\left(t_{n+1}\right) h M\right]\left(1+\frac{h}{2} k\left(t_{n}\right)+k\left(t_{n+1}\right)\right) \\
& +\frac{h}{2}\left[2 w(h)+h k\left(t_{n-1}\right) M+h k\left(t_{n}\right) M-K\left(t_{n}\right) h M\right] \\
& =\left\|e_{0}\right\| \prod_{i=0}^{n}\left(1+\frac{h}{2} k\left(t_{n-i}\right)+k\left(t_{n-(i-1)}\right)\right)+\frac{h}{2}\left[2 w(h)+h k\left(t_{n-2}\right) M+h k\left(t_{n-1}\right) M-K\left(t_{n-1}\right) h M\right] \\
& \times \prod_{i=0}^{n-1}\left(1+\frac{h}{2} k\left(t_{n-i}\right)+k\left(t_{n-(i-1)}\right)\right)+\frac{h}{2}\left[2 w(h)+h k\left(t_{n-1}\right) M+h k\left(t_{n}\right) M-K\left(t_{n}\right) h M\right] \\
& \times \prod_{i=0}^{n-2}\left(1+\frac{h}{2} k\left(t_{n-i}\right)+k\left(t_{n-(i-1)}\right)\right)+\cdots+\frac{h}{2}\left[2 w(h)+h k\left(t_{n}\right) M+h k\left(t_{n+1}\right) M-K\left(t_{n+1}\right) h M\right] \\
& \times\left(1+\frac{h}{2} k\left(t_{n}\right)+k\left(t_{n+1}\right)\right)+\frac{h}{2}\left[2 w(h)+h k\left(t_{n-1}\right) M+h k\left(t_{n}\right) M-K\left(t_{n}\right) h M\right] \\
& =\left\|e_{0}\right\| \prod_{i=0}^{n}\left(1+\frac{h}{2} k\left(t_{n-i}\right)+k\left(t_{n-(i-1)}\right)\right)+\frac{h}{2}\left[2 w(h)+h k\left(t_{n-2}\right) M+h k\left(t_{n-1}\right) M-K\left(t_{n-1}\right) h M\right] \\
& \times\left[1+\prod_{i=0}^{n-1}\left(1+\frac{h}{2} k\left(t_{n-i}\right)+k\left(t_{n-(i-1)}\right)\right)+\prod_{i=0}^{n-2}\left(1+\frac{h}{2} k\left(t_{n-i}\right)+k\left(t_{n-(i-1)}\right)\right)+\cdots+\left(1+\frac{h}{2} k\left(t_{n}\right)+k\left(t_{n+1}\right)\right)\right] .
\end{aligned}
$$

Taking into account that $e_{0}=0$ where $e_{0}=X_{0}-X\left(t_{0}\right)=0$ and by taking the limit as: 


$$
h \rightarrow 0 \text { then we have: } \lim _{h \rightarrow 0}\left\|e_{n+1}\right\| \rightarrow 0
$$

i.e. $\left\{e_{n}\right\}$ converge in m.s. to zero as: $h \rightarrow 0$ hence $X_{n} \stackrel{\text { m.s }}{\longrightarrow} X\left(t_{n}\right)=X(t)$.

\section{Case Study}

Example: Solve the problem

$$
\frac{\mathrm{d} N}{\mathrm{~d} t}=\alpha N, \quad N(0)=1000, t \in[0,1], \quad \alpha \sim \text { random variable. }
$$

The theoretical solution is:

$$
N(t)=1000 \mathrm{e}^{0.5 \alpha}
$$

\begin{tabular}{|c|c|c|c|c|c|}
\hline \multicolumn{6}{|c|}{$\alpha \sim \operatorname{Binomial}(5,0.2)$} \\
\hline$h$ (step size) & $\begin{array}{l}E[y] \text { for the exact } \\
\text { solution }\end{array}$ & $\begin{array}{c}E\left[y_{n}\right] \text { for Heun's } \\
\text { method }\end{array}$ & Error on Heun's & Error on Euler [12] & $\begin{array}{c}\text { Error on Runge } \\
\text { Kutta [12] }\end{array}$ \\
\hline 0.25 & 1318.179242 & 1306.250000 & 11.929242 & 68.179242 & 156.820758 \\
\hline 0.125 & 1140.431227 & 1128.515625 & 1.368727 & 15.431227 & 97.068773 \\
\hline 0.025 & 1025.572765 & 1025.562500 & 0.010265 & 0.572765 & 21.927235 \\
\hline 0.0025 & 1002.505635 & 1002.505625 & 0.000010 & 0.005635 & 2.244365 \\
\hline \multicolumn{6}{|c|}{$\alpha \sim$ Erlang $(0.5,2)$} \\
\hline 0.25 & 1306.122449 & 1296.875000 & 9.247449 & 56.122449 & 131.377551 \\
\hline 0.125 & 1137.777778 & 1136.718750 & 1.059028 & 12.777778 & 80.972222 \\
\hline 0.025 & 1025.572765 & 1025.468750 & 0.007936 & 0.476686 & 18.273314 \\
\hline 0.0025 & 1002.505635 & 1002.504688 & 0.000007 & 0.004695 & 1.870305 \\
\hline \multicolumn{6}{|c|}{$\alpha \sim$ Poisson $(2)$} \\
\hline 0.25 & 1764.823762 & 1687.500000 & 77.323762 & 264.823762 & 485.176238 \\
\hline 0.125 & 1305.122500 & 1296.875000 & 8.247500 & 55.122500 & 319.877500 \\
\hline 0.025 & 1051.933860 & 1051.875000 & 0.058860 & 1.933860 & 73.066140 \\
\hline 0.0025 & 1005.018808 & 1005.018750 & 0.000058 & 0.018808 & 7.481192 \\
\hline
\end{tabular}

The approximations:

In this results showed in the table we have the Heun's method gave better approximation as: $h \rightarrow 0$ than Euler and Rung-Kutta [12] for solving random variable, initial value problems.

\section{Conclusion}

The initially valued first order random differential equations can be solved numerically using the random Heun's methods in mean square sense. The convergence of the presented numerical techniques has been proven in mean square sense. The results of the paper have been illustrated through an example.

\section{References}

[1] Burrage, K. and Burrage, P.M. (1996) High Strong Order Explicit Runge-Kutta Methods for Stochastic Ordinary Differential Equations. Applied Numerical Mathematics, 22, 81-101. http://dx.doi.org/10.1016/S0168-9274(96)00027-X

[2] Burrage, K. and Burrage, P.M. (1998) General Order Conditions for Stochastic Runge-Kutta Methods for Both Commuting and Non-Commuting Stochastic Ordinary Equations. Applied Numerical Mathematics, 28, 161-177. 
http://dx.doi.org/10.1016/S0168-9274(98)00042-7

[3] Cortes, J.C., Jodar, L. and Villafuerte, L. (2007) Numerical Solution of Random Differential Equations, a Mean Square Approach. Mathematical and Computer Modeling, 45, 757-765. http://dx.doi.org/10.1016/j.mcm.2006.07.017

[4] Cortes, J.C., Jodar, L. and Villafuerte, L. (2006) A Random Euler Method for Solving Differential Equations with Uncertainties. Progress in Industrial Mathematics at ECMI.

[5] Lamba, H., Mattingly, J.C. and Stuart, A. (2007) An Adaptive Euler-Maruyama Scheme for SDEs, Convergence and Stability. IMA Journal of Numerical Analysis, 27, 479-506. http://dx.doi.org/10.1093/imanum/drl032

[6] Platen, E. (1999) An Introduction to Numerical Methods for Stochastic Differential Equations. Acta Numerica, 8, 197-246. http://dx.doi.org/10.1017/S0962492900002920

[7] Higham, D.J. (2001) An Algorithmic Introduction to Numerical Simulation of SDE. SIAM Review, 43, 525-546. http://dx.doi.org/10.1137/S0036144500378302

[8] Talay, D. and Tubaro, L. (1990) Expansion of the Global Error for Numerical Schemes Solving Stochastic Differential Equation. Stochastic Analysis and Applications, 8, 483-509. http://dx.doi.org/10.1080/07362999008809220

[9] Burrage, P.M. (1999) Numerical Methods for SDE. Ph.D. Thesis, University of Queensland, Brisbane.

[10] Kloeden, P.E., Platen, E. and Schurz, H. (1997) Numerical Solution of SDE through Computer Experiments. 2nd Edition, Springer, Berlin.

[11] El-Tawil, M.A. (2005) The Approximate Solutions of Some Stochastic Differential Equations Using Transformation. Applied Mathematics and Computation, 164, 167-178. http://dx.doi.org/10.1016/j.amc.2004.04.062

[12] El-Tawil, M.A. and Sohaly, M.A. (2011) Mean Square Numerical Methods for Initial Value Random Differential Equations. Open Journal of Discrete Mathematics, 1, 66-84. http://dx.doi.org/10.4236/ojdm.2011.12009

[13] Kloeden, P.E. and Platen, E. (1999) Numerical Solution of Stochastic Differential Equations. Springer, Berlin. 
Scientific Research Publishing (SCIRP) is one of the largest Open Access journal publishers. It is currently publishing more than 200 open access, online, peer-reviewed journals covering a wide range of academic disciplines. SCIRP serves the worldwide academic communities and contributes to the progress and application of science with its publication.

Other selected journals from SCIRP are listed as below. Submit your manuscript to us via either submit@scirp.org or Online Submission Portal.
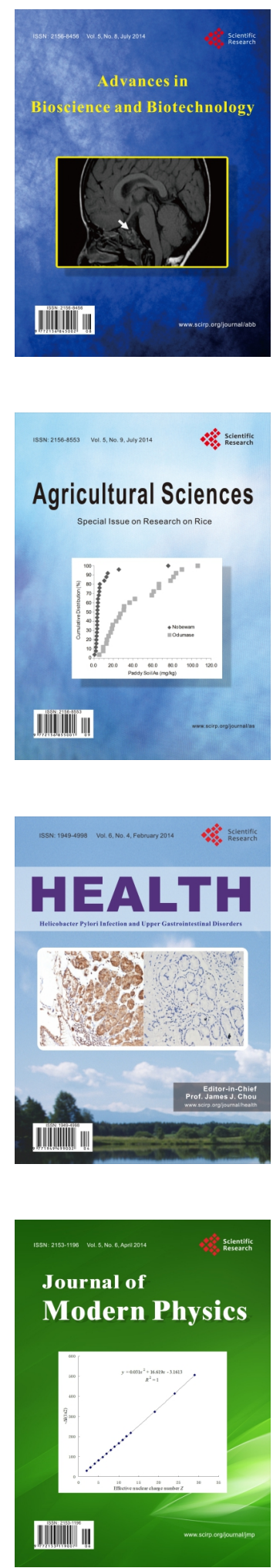
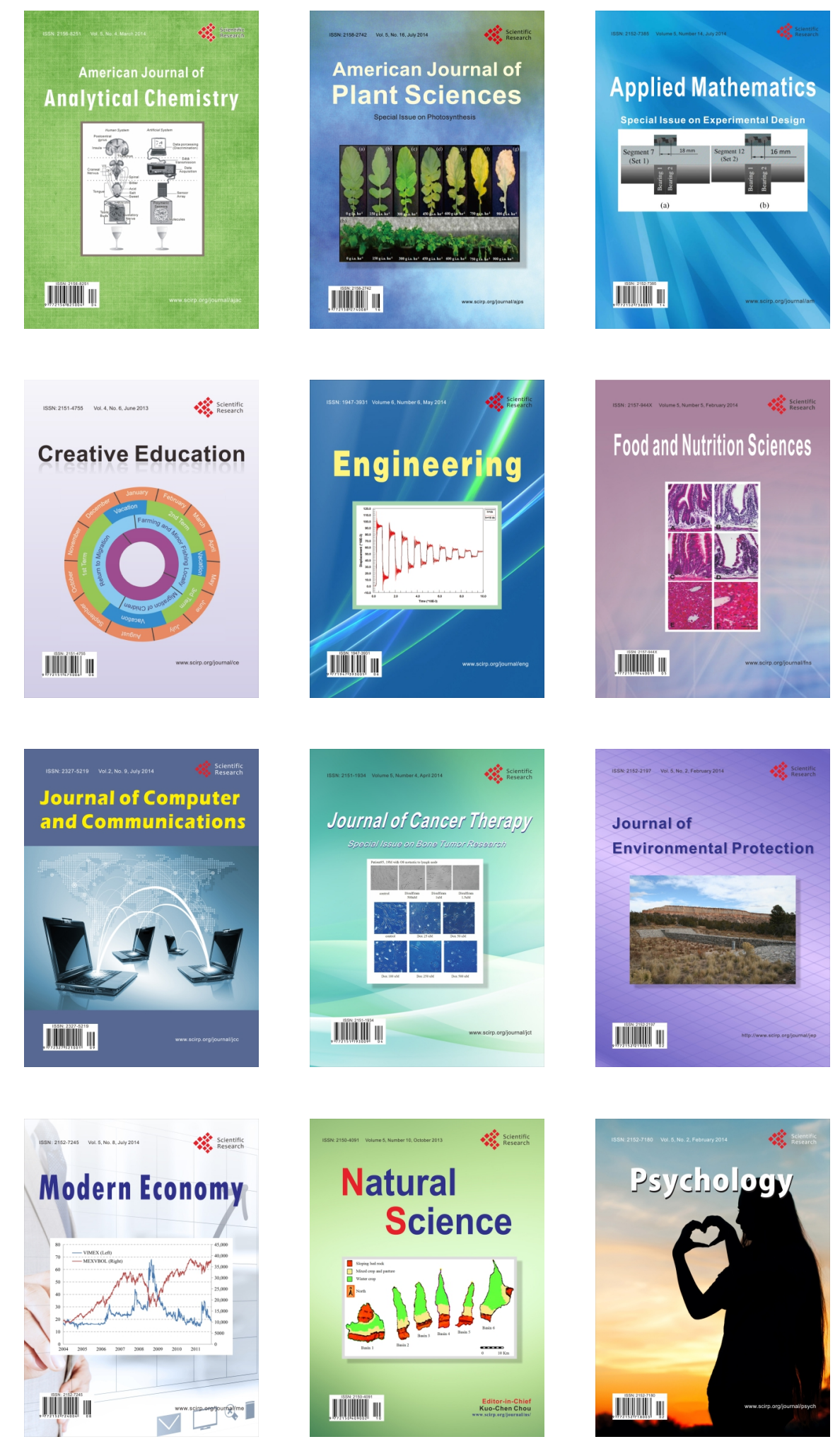